\title{
A Novel Oversampling Technique Based on the Manifold Distance for Class Imbalance Learning
}

\section{Yinan Guo, Botao Jiao and Lingkai Yang}

School of Information and Control Engineering,

China University of Mining and Technology,

Xuzhou, China

E-mail: nanfly@126.com, 461375470@qq.com, yanglk@cumt.edu.cn

\section{Jian Cheng*}

China Coal Research Institute,

Beijing 100013, China

E-mail: chengjian@cumt.edu.cn

*Corresponding author

\section{Shengxiang Yang}

De Montfort University

Leicester LE1 9BH, United Kingdom

E-mail:syang@dmu.ac.uk

\section{Fengzhen Tang}

Shenyang Institute of Automation

Shenyang, China

E-mail:tangfengzhen@sia.cn

\begin{abstract}
Oversampling is a popular problem-solver for class imbalance learning by generating more minority samples to balance the dataset size of different classes. However, resampling in original space is ineffective for the imbalance datasets with class overlapping or small disjunction. Based on this, a novel oversampling technique based on manifold distance is proposed, in which a new minority sample is produced in terms of the distances among neighbors in manifold space, rather than the Euclidean distance among them. After mapping the original data to its manifold structure, the overlapped majority and minority samples will lie in areas easily being partitioned. In addition, the new samples are generated based on the neighbors locating nearby in manifold space, avoiding the adverse effect of the disjoint minority classes. Following that, an adaptive adjustment method is presented to determine the number of the newly generated minority samples according to the distribution density of the matched-pair data. The experimental results on 48 imbalanced datasets indicate that the proposed oversampling technique has the better classification accuracy.
\end{abstract}

Keywords: class imbalance learning; oversampling; manifold learning; overlapping; small disjunction.

Reference to this paper should be made as follows: Guo, Y., Jiao, B., Yang, L., Cheng, J., Yang, S., and Tang, F. $(\mathrm{xxxx})$ 'A Novel Oversampling Technique Based on the Manifold Distance for Class Imbalance Learning', International Journal of Bio-Inspired Computation, Vol. x, No. x, pp.xxx-xxx.

Biographical notes: Yinan Guo is a Professor in China University of Mining and Technology. Her research interests include class imbalance learning, computation intelligence in dynamic and uncertain optimization and their applications.

Jian Cheng is a associate professor in China Coal Research Institute. His research interests include imbalance learning and image processing.

Shengxiang Yang is a Professor in De Montfort University. His research interests include computation intelligence in dynamic and uncertain optimization and their applications.

Fengzhen Tang is an Assistant Researcher in Shenyang Institute of Automation. Her research interests include machine learning and computational neuroscience. 


\section{Introduction}

The distribution of data is skewed in many real-world classification problems, such as fault diagnosis (Cai et al., 2017), face recognition (Soleymani et al., 2018), and fraud detection (Dal et al., 2018). That is, more data belongs to the majority class. However, the events corresponding to the minority samples, such as a failure of a space-shuttle (Shakeel et al., 2017), may result in a huge product cost. Hence, to accurately label the data in the minority class attracts the increasing interest. In particular, the small data size of the minority samples provide the limited information for class imbalance learning, causing the wrong classification boundary. Moreover, the implicit problems like class overlapping and small disjunction also bring severe hindrance to the performance of a classifier (Krawczyk, 2016). The crossdistributed minority and majority samples easily make the classifier overfitting or underfitting. The imbalanced data with small disjunction contains several minority subclusters lying far away each other, forming the complicated classification boundary.

Previous studies on class imbalance learning can be categorized into data-, algorithm-, or hybrid-level strategies (Loezer et al., 2020; Kang et al., 2018; Wang et al., 2016; Cheng et al., 2019). Among them, data-level strategies balance the distribution of imbalanced dataset by oversampling the minority samples or undersampling the majority samples. Synthetic minority oversampling technique (SMOTE) (Arafat et al., 2019) created the new synthetic samples along the line between a minority sample and its nearest neighbors. Following that, many improved SMOTE-based algorithms were proposed. Borderlinesynthetic minority oversampling technique (BorderlineSMOTE)(Han et al., 2005) only oversampled the minority samples nearby the classification boundary. Adaptive synthetic sampling approach(ADASYN) (He et al., 2008) and majority-weighted minority oversampling technique (MWMOTE) (Barua et al., 2013) both assigned the weights for the hard-to-learn minority samples in terms of the Euclidean distances to the nearest majority samples, and then generated a new minority sample in any minority sub-cluster. In order to identify the data distribution with small disjunction in original space, some clustering-based data-level methods were presented (Lin et al., 2017). Lim (Lim et al., 2016) proposed evolutionary cluster-based synthetic oversampling ensemble (ECO-Ensemble), in which the clustering methods was introduced to oversample the synthetic minority samples in each sub-cluster. However, the above oversampling methods generate the minority samples in terms of the data structure in original space, which may be labelled incorrectly, especially for the imbalanced data with class overlapping and small disjunction.

Manifold learning maps high-dimensional data into the low-dimensional manifold space, which makes the

Copyright @ 201X Inderscience Enterprises Ltd. cross-distributed imbalanced data in original space more easily being partitioned and creates the more qualified samples. Moreover, for the minority samples lying in disjoint sub-areas, a new sample created along the line connecting these sub-areas in original space may be incorrectly labelled. Different from it, the minority samples that produce based on the neighbors chosen from the disjoint sub-area by their manifold distances are close to one of the minority sub-clusters. Apparently, the manifold distance is good for retaining the original structure of the imbalanced data and provides a rational criterion for finding the rational neighbors of the minority samples lying in overlapping or disjoint area. Based on this, Bellinger, et al (Bellinger et al., 2017) found the neighbors in manifold space, and then mapped them back to original space. The new instances, subsequently, were generated by oversampling technique and employed to classify the highdimensional imbalanced datasets. However, the forward and backward mapping between manifold space and original space increases the computational cost.

To address the problems, a novel oversampling technique based on the manifold distance (OTEMD) is proposed to produce a new minority sample in terms of the distances among the neighbors in manifold space. In order to decrease the computation complexity for extracting the neighbors, the redundant majority samples are firstly removed by undersampling. Following that, the nearest samples are selected as the neighbors in terms of their manifold distances, and finally the new minority samples are generated based on the matchedpair data by oversampling. In general, no matter how dense the matched-pair data is, the same number of the new samples are generated by oversampling, which makes the difference of the data density among the samples more serious, causing the weak classification performance in the sparse areas. Thus, a distance mechanism is introduced to produce more samples for the matched-pair data with the sparse distribution and close to the classification boundary. Based on this, four kinds of oversampling strategies derived from borderlineSMOTE-1 or borderline-SMOTE-2, expressed by $O S_{1}$, $O S_{2}, O S_{3}$ and $O S_{4}$, are presented, with the purpose of drawing the more accurate classification lines with the less computation complexity.

\section{An Oversampling Technique Based On The Manifold Distance}

OTEMD provides a generic framework for generating a synthetic minority sample on the basis of the neighbors selected by their manifold distance. Three key issues are undersampling, extracting the nearest neighbors based on manifold distance, and oversampling. Undersampling strategy is implemented before the data-mapping process, with the purpose of reserving more representative majority samples and reducing the computation complexity of manifold learning. After 
Figure 1: Flowchart of OTEMD

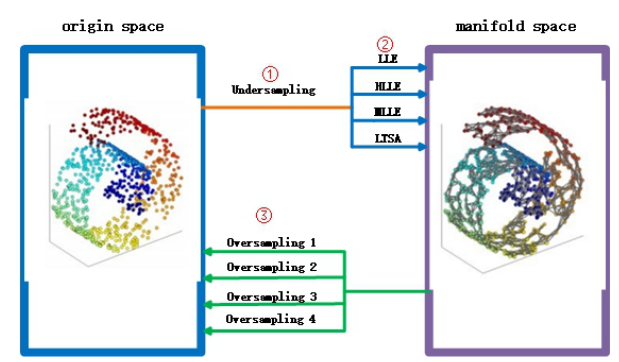

mapping the samples from original space to manifold space, the neighbors of each minority sample are selected to build the matched-pair data in terms of the manifold distance among the samples. Following that, a new minority sample is generated between the minority one and its nearest neighbors.

\subsection{Undersampling}

The goal of undersampling is to remove the data with redundant or useless information from the majority class. A valuable sample is the one that belongs to the different class or has the sparse distribution with its neighbors. To this end, a majority sample is removed if its neighbors all belong to the majority class and distribute densely.

Suppose the original imbalanced dataset expressed by $O$ contains $n_{l}$ majority samples and $n_{s}$ minority data. The totally data size satisfies $n_{o}=n_{l}+n_{s}$. For each sample, the average distance between it and $k_{1}$ neighbors is called the distribution density, denoted as $d_{i}$. Let $o_{i}$ and $o_{i p}$ be $i$ th sample and its $p$ th neighbor.

$$
d_{i}=\frac{1}{k_{1}} \sum_{p=1}^{k_{1}}\left\|o_{i}-o_{i p}\right\|_{2}
$$

Let $d=\frac{1}{n_{o}} \sum_{i=1}^{n_{o}} d_{i}$ be the average distribution density for all samples, and $\alpha \in(0,1)$ is a preset constant. The distribution of samples is dense if they are close to their neighbors. Based on this, the majority samples satisfying $d_{i}<d * \alpha$ are considered as the redundant ones.

Denote $u_{i}$ and $y_{i}^{u}$ are $i$ th sample and its label after undersampling. The dataset after undersampling expressed by $U$ is composed of $u_{l}$ majority samples and $u_{s}$ minority ones, and its data size is $n_{u}$. The detailed algorithm steps of undersampling are shown as Algorithm 1.

\subsection{Extracting the Neighbors Based on the Manifold Distance}

Manifold learning focuses on the low-dimensional structure embedded in the original dataset (Lunga et al., 2013). After mapping the original data to manifold space, the manifold structure is explored and the neighbors are extracted to build the matched-pair data for generating the new minority samples. Based on this,

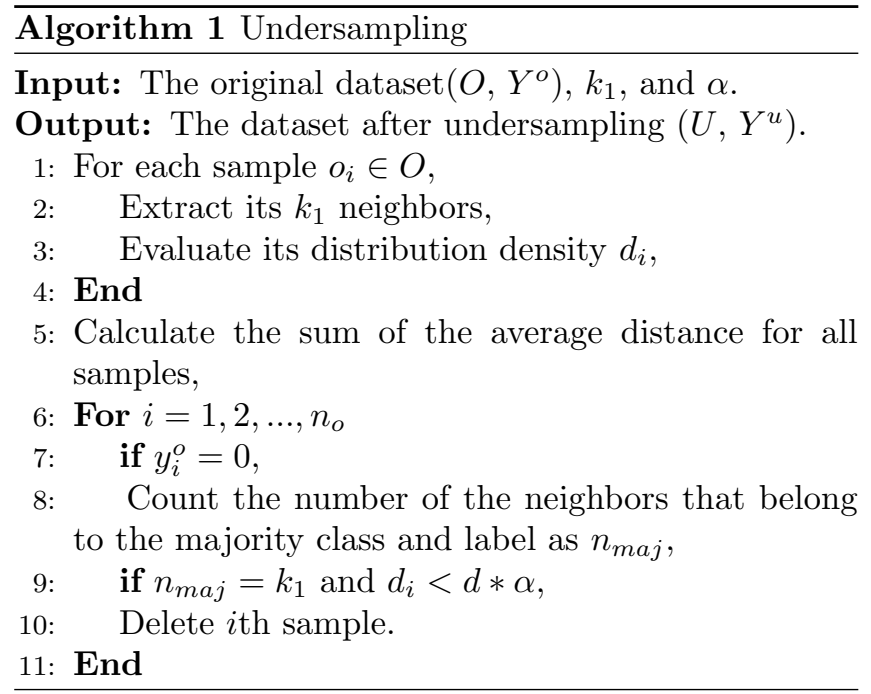

four manifold learning methods, including locally linear embedding (LLE), Hessian-based LLE (HLLE), modified LLE (MLLE), and local tangent space alignment (LTSA) (Zhang et al., 2018), are employed to map the original imbalanced data to manifold space. Among them, LLE found the manifold embedding by preserving the distances unchanged within the neighbors of each sample (Roweis et al., 2000). Different from it, MLLE (Shen et al., 2016) and HLLE employed multiple weights and Hessian-based quadratic to address the regularization. LTSA prefers to find the embedding that aligns in the tangent space. In this following part, LLE that employed in OTEMD is illustrated in detail.

At first, each sample is reconstructed by its nearest neighbors, which has the minimum error $\varepsilon(W)$. Assume that there are $k_{2}$ neighbors. $w_{i j}$ represents the relationship between $i$ th sample and its neighbors, satisfying $\sum_{j}^{k_{2}} w_{i j}=1 . w_{i j}=0$ if $u_{j}$ isn't the nearest neighbor of $u_{i}$.

$$
\varepsilon(W)=\sum_{i=1}^{n_{u}}\left(u_{i}-\sum_{j}^{k_{2}} w_{i j} u_{j}\right)^{2}
$$

Suppose $M$ is the dataset in manifold space. Under the obtained $W$, the manifold structure is explored by minimizing $\phi(M)=\sum_{i=1}^{n_{u}}\left(m_{i}-\sum_{j}^{k_{2}} w_{i j} m_{j}\right)^{2}$. Based on $M$, the matched-pair data are built by exploring the neighbors of each minority sample. The detailed algorithm steps of extracting the neighbors in manifold space are shown in Algorithm 2.

For an imbalanced dataset, the overlapping samples that belong to different classes and locate closely may generate the new samples incorrectly labelled, resulting in the inaccurate classification boundary. To solve the problem, manifold learning that projects the original data to the low-dimensional space is introduced to find the more easily partitioned manifold structure for the inseparable data by the kernel trick in SVM or principal component analysis (Kang et al., 2014). In addition, the samples generated by two minority sub-clusters lying in the disjoint areas are normally grouped into 


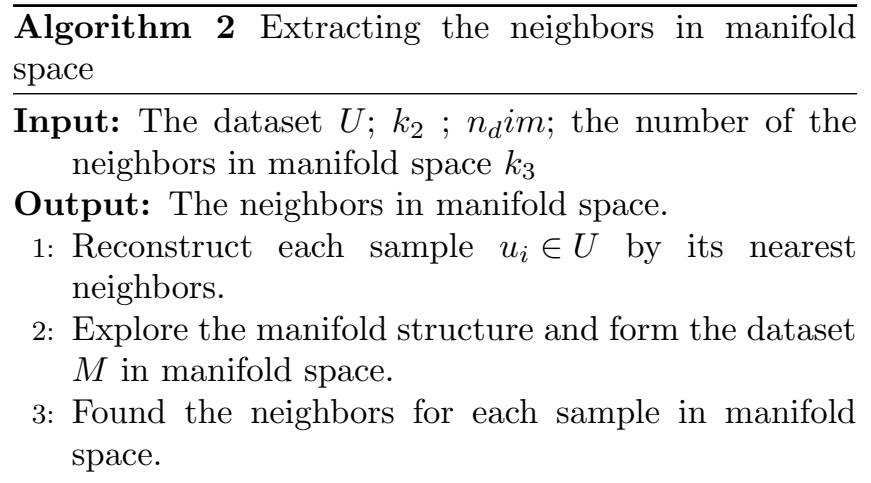

the minority class. However, they may be mislabeled because the neighbors that selected in terms of the shortest Euclidean distance are located in the irrelevant areas. After reconstructing the data in manifold space, two nearest minority samples in the disjoint areas of original space may be located far away in manifold space. Therefore, only the minority samples belonging to the same sub-cluster can be selected as the neighbors in terms of the manifold distance.

\subsection{Oversampling}

Given that a minority sample far away from the classification boundary helps nothing to improve the accuracy, a distance mechanism is introduced to form four improved over-sampling strategies based on Borderline-SMOTE. To our best of knowledge, Borderline-SMOTE produces a minority sample based on the matched-pair data near the classification boundary, and the equal number of new samples are created for each matched-pair data. However, more samples may be generated from the neighbors with the dense distribution, which provide redundant, even useless information for the classification, and consume the extra computation resource. Thus, we present a distance mechanism to produce more samples in the sparse areas for the matched-pair data far away from each other, with the purpose of drawing the more accurate classification boundary.

Borderline-SMOTE-1 generates a sample from the minority matched-pair data near the classification boundary in original space. To address class overlapping and small disjunction, the neighbors are selected in terms of their manifold distances. Thereafter, a novel oversampling strategy based on Borderline-SMOTE-1, denoted as $O S_{1}$, is presented to create the same amount of minority samples for each minority matched-pair data. Taking the distribution density of the neighbors into account, $O S_{3}$ oversampling strategy produces less minority samples from the matched-pair data lying in the dense area. Different from them, $O S_{2}$ and $O S_{4}$ derived from Borderline-SMOTE-2 generate a new sample from not only the minority matched-pair data, but also the minority-majority ones. More specially, the minoritymajority matched-pair data is employed only if the ratio of minority samples to their neighbors is larger than half of the imbalance ratio.

Suppose $I R=\frac{u_{l}}{u_{s}}$ is the imbalance ratio of a dataset and $g=u_{l}-u_{s}$ is the number of the generated minority samples. Let $n_{m a j}$ be the number of neighbors belonging to the majority class and $n_{G}$ is the number of the matched-pair data. A minority sample satisfying $n_{m a j} \in$ $\left[0.5 k_{3}, k_{3}\right)$ is considered as a valuable one near the classification boundary. The corresponding matchedpair data obtained based on the manifold distance is employed to produce a new minority sample. For $O S_{1}$ and $O S_{2}$, the number of the generated minority ones is same for all matched-pair data, expressed by $n_{n e w}$.

$$
n_{\text {new }}=\frac{g}{n_{G}}
$$

$O S_{3}$ and $O S_{4}$ generate the different number of the minority samples in terms of the distribution density of the matched-pair data, denoted as $p_{i}$. Let $d_{i}^{m}$ be the manifold distances of the matched-pair data for $i$ th minority sample.

$$
p_{i}=\frac{d_{i}^{m}}{n_{G}} \sum_{i=1}^{n_{G}}\left(d_{i}^{m}\right)
$$

There are $g$ minority sample are generated by randomly sampling with replacement for all matchedpair data in terms of $p_{i}$. Because the matched-pair data with long distance is easier to be selected multiple times, more minority samples will be generated around them.

\section{Experimental Results And Discussion}

Nine synthetic and thirty-nine imbalanced datasets derived from the UCI machine learning repository are employed to compare the classification performances among SMOTE, Borderline-SMOTE, ADASYN, MWMOTE, ACOSampling, and the proposed oversampling strategy. Decision tree(DT) is used as the basic classifier. To synthetically evaluate the performances of class imbalance algorithms, Accuracy, G-mean, F-measure and the area under the curve (AUC) are employed as the metrics (Lim et al., 2016). Moreover, Wilcoxon paired signed-rank test is carried out to determine whether the proposed method is significantly better than others or not.

\subsection{The classification performances on the synthetic Dataset}

In order to intuitively compare the oversampling performance of various methods on the imbalanced dataset with small disjunction, 30 randomly generated samples are mixed with the Swiss-roll dataset, which simulate the real-world imbalanced dataset with noise and small disjunction. Fig.2(a) depicts the distribution of the mixed dataset. The red and blue points represent the majority and minority samples in original space, 
respectively. The distribution of all samples in original space by various oversampling strategies are shown in Fig.2(b)-(j). Owing to the noise, some samples that newly developed by SMOTE, Borderline-SMOTE-1, and Borderline-SMOTE-2 may lie outside of the surface along the Swiss-roll curve. Apparently, all methods form the satisfied Swiss-roll after oversampling, except for SMOTE because it generates a new sample on the basis of the nearest minority samples by linear interpolation regardless of the structure of the whole dataset, resulting in the unexpected minority samples. More specially, the more appropriate neighbors can be found by four kinds of OTEMD-LLE in terms of the manifold distance and help to generate the satisfied samples that meet the need of the original data.

Figure 2: The performances of different oversampling techniques for the Swiss-roll dataset

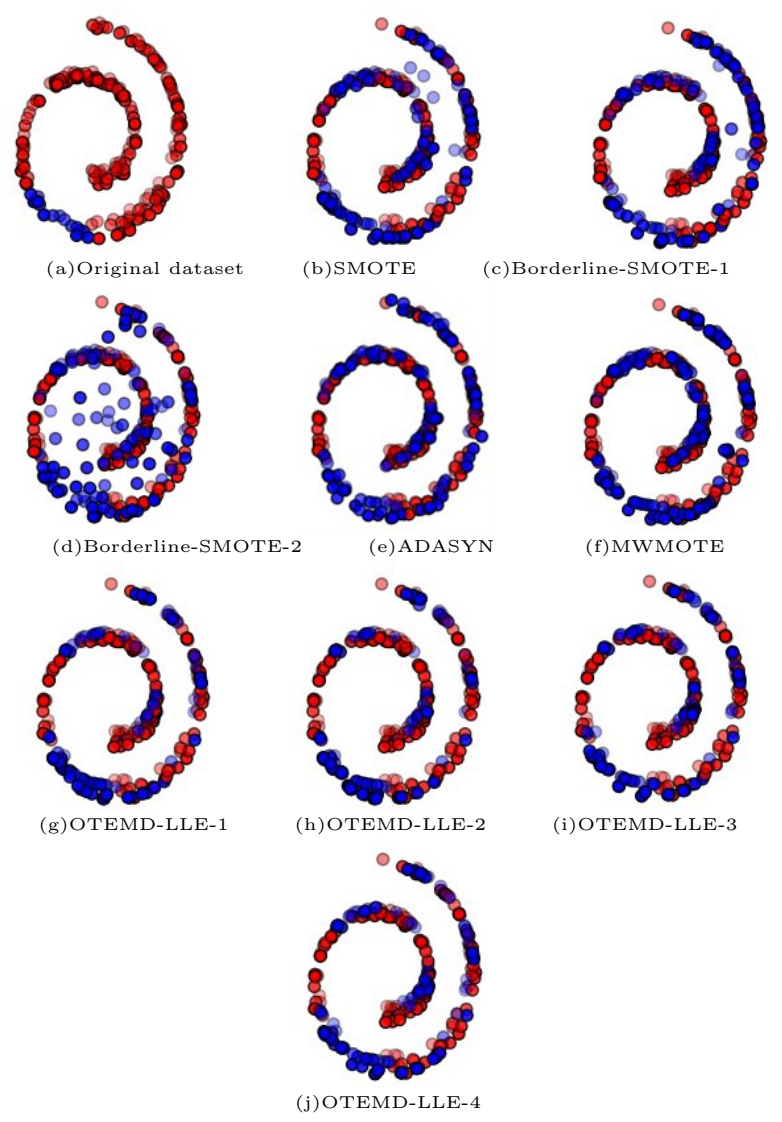

Nine imbalanced datasets composed of the samples satisfying the normal distribution are constructed to further analyze the classification performance of OTEMD. The dataset contains two disjoint minority sub-clusters, namely, Min-A and Min-B. The margin between Min-A and the majority class retains $[0,1,1]$ and $[0,0,0]$, whereas the margin changes for Min-B. As shown in Table 1, IR and M_B represent the imbalance ratio and the margin between the Min-B and majority class, respectively. The AUC values obtained by different oversampling techniques on the artificial datasets shown in Figure.3 indicate that OTEMD-based methods have the superior classification accuracy. In particular, OTEMD-LLE-1 achieves the largest average AUC on the artificial datasets, as shown in Table 2.

Table 1. The attributes of synthetic datasets

\begin{tabular}{cccccc}
\hline Case & IR & Maj & Min-A & Min-B & M_B \\
\hline C1 & 1.76 & 300 & 150 & 20 & $0,2,2$ \\
C2 & 1.76 & 300 & 150 & 20 & $0,1.5,1.5$ \\
C3 & 1.76 & 300 & 150 & 20 & $0,1.25,1.25$ \\
C4 & 2.91 & 350 & 100 & 20 & $0,2,2$ \\
C5 & 2.91 & 350 & 100 & 20 & $0,1.5,1.5$ \\
C6 & 2.91 & 350 & 100 & 20 & $0,1.25,1.25$ \\
C7 & 5.71 & 400 & 50 & 20 & $0,2,2$ \\
C8 & 5.71 & 400 & 50 & 20 & $0,1.5,1.5$ \\
C9 & 5.71 & 400 & 50 & 20 & $0,1.25,1.25$ \\
\hline
\end{tabular}

\subsection{The classification performances on UCI Datasets}

Thirty-nine datasets with the various distribution and the imbalance ratio are chosen from the UCI machine learning repository and employed to fully compare and analyze the classification performance of different oversampling techniques. However, several datasets are multiclass imbalance classification problems. Thus, we transformed them into binary-classification problems by combining the data in most of the classes into majority samples. In the experiments, the number of nearest neighbors for SMOTE, Borderline-SMOTE and ADASYN are set to $10 . k_{1}, k_{2}$ and $k_{3}$ of MWMOTE are set to 5, 3 and 0.5, respectively. The number of ants and the terminal iterations for ACOSampling are both 10 , and the evaporation coefficient is 0.8 . The hyperparameters in OTEMD satisfy $k_{1}=k_{2}=k_{3}$, and the optimal $k_{2}$ and $n_{d} i m$ are chosen from $[10,15,20,25]$ and $[3,5,7]$ by three-fold cross validation.

For OTWMD, the mapping method from original space to manifold space, as well as the oversampling strategies are the key issues. To further analyze their impact on the classification performances, F-measure, Gmean and AUC of different oversampling strategies are compared in Tables 3, 4 and 5, respectively. Apparently, the proposed oversampling strategies outperform the others for most of the imbalanced datasets, especially for the ones with higher imbalance ratio. More specially, OTEMD-LLE-2 obtains the best average AUC, whereas OTEMD-LLE-1 has the superior F-mean. By compared the performances of OTEMD with various mapping methods, including LLE, HLLE, MLLE, and LSTA, shown in Table 6, we see that the new minority samples generated by $O S_{2}$ provide more valuable information for the classification boundary.

Wilcoxon paired sign-rank test is applied to pairwise compare the performances of the proposed OTEMD method with the others on 39 datasets. The null hypothesis of Wilcoxon test $H_{0}$ indicates that OTEMD has the same classification accuracy as the other algorithms, and the alternative hypothesis $H_{\alpha}$ shows the significantly different performances between them. 
Figure 3: The performances of different oversampling techniques for the artificial datasets

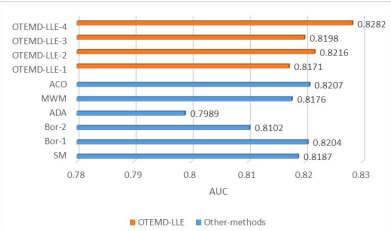

C1

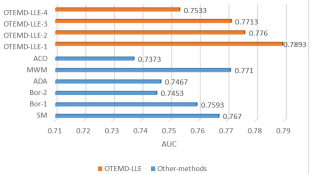

C5

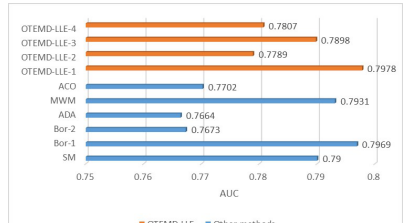

C2

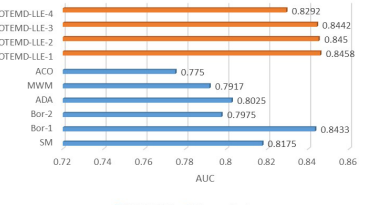

C3

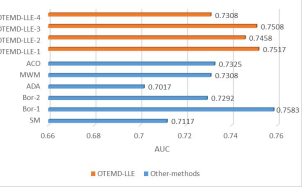

C8

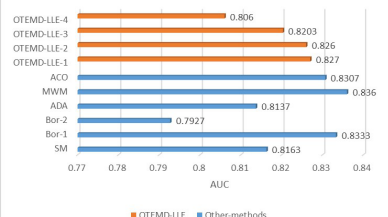

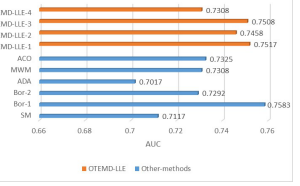

C6

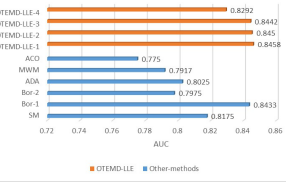

C7
C4

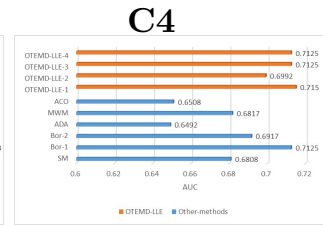

C9

Table 2. The average AUC of different algorithms

\begin{tabular}{ccccccccccc}
\hline & SM & Bor-1 & Bor-1 & ADA & MWM & ACO & OTEMD-LLE-1 & OTEMD-LLE-2 & OTEMD-LLE-3 & OTEMD-LLE-4 \\
\hline Average & 0.7701 & 0.7917 & 0.7622 & 0.7537 & 0.7716 & 0.7583 & $\mathbf{0 . 7 9 3 4}$ & 0.7870 & 0.7893 & 0.7779 \\
\hline Rank & 7 & 2 & 8 & 10 & 6 & 9 & $\mathbf{1}$ & 4 & 3 & 5 \\
\hline
\end{tabular}

Table 3. Comparison of the AUC among different oversampling techniques

\begin{tabular}{|c|c|c|c|c|c|c|c|c|c|c|}
\hline Dataset & SM & Bor-1 & Bor-2 & ADA & MWM & $\mathrm{ACO}$ & OTEMD-LLE-1 & OTEMD-LLE-2 & OTEMD-LLE-3 & OTEMD-LLE-4 \\
\hline Abalone_18v9 & 0.6590 & 0.6936 & 0.6538 & 0.6115 & 0.6250 & 0.6160 & 0.6609 & 0.7346 & 0.6571 & 0.6500 \\
\hline Abalone_17v7 & 0.9429 & 0.9474 & 0.9191 & 0.9583 & 0.9409 & 0.9685 & 0.9288 & 0.9089 & 0.8794 & 0.9134 \\
\hline Abalone_19v5 & 1.0000 & 1.0000 & 0.9867 & 0.9933 & 0.9867 & 0.9867 & 0.9933 & 0.9933 & 0.9933 & 0.9933 \\
\hline CTG_PvN & 0.9589 & 0.9643 & 0.9710 & 0.9649 & 0.9650 & 0.9561 & 0.9561 & 0.9721 & 0.9708 & 0.9825 \\
\hline CTG_SvN & 0.9492 & 0.9587 & 0.9464 & 0.9475 & 0.9313 & 0.9492 & 0.9545 & 0.9522 & 0.9502 & 0.9467 \\
\hline Statlandsat_4v12 & 0.9746 & 0.9789 & 0.9732 & 0.9889 & 0.9775 & 0.9579 & 0.9846 & 0.9816 & 0.9761 & 0.9802 \\
\hline Statlandsat_5v12 & 0.9697 & 0.9767 & 0.9586 & 0.9731 & 0.9681 & 0.9611 & 0.9689 & 0.9703 & 0.9589 & 0.9783 \\
\hline Libra_123vAll & 0.8758 & 0.9167 & 0.8840 & 0.9096 & 0.9069 & 0.9012 & 0.9122 & 0.9055 & 0.9258 & 0.8711 \\
\hline Libra_456vAll & 0.9524 & 0.9557 & 0.9620 & 0.9622 & 0.9694 & 0.9182 & 0.9543 & 0.9505 & 0.9301 & 0.9361 \\
\hline Yeast_ME1vCYT & 1.0000 & 0.9984 & 0.9770 & 1.0000 & 0.9770 & 0.9841 & 0.9929 & 0.9984 & 0.9929 & 0.9929 \\
\hline Yeast_ME1vNUC & 0.9673 & 0.9602 & 0.9673 & 0.9531 & 0.9714 & 0.9714 & 0.9786 & 0.9786 & 0.9786 & 0.9908 \\
\hline Yeast_ME2vCYT & 0.9498 & 0.9332 & 0.8869 & 0.9498 & 0.9782 & 0.9564 & 0.9616 & 0.9253 & 0.9723 & 0.9766 \\
\hline Yeast_ME2vNUC & 0.9141 & 0.9121 & 0.8937 & 0.9161 & 0.9373 & 0.9380 & 0.9646 & 0.9666 & 0.9555 & 0.9514 \\
\hline Yeast_ME3vCYT & 0.9473 & 0.9489 & 0.9227 & 0.9371 & 0.9379 & 0.9556 & 0.9454 & 0.9624 & 0.9585 & 0.9208 \\
\hline Yeast_ME3vNUC & 0.9349 & 0.9169 & 0.9267 & 0.9320 & 0.9190 & 0.9364 & 0.9114 & 0.8986 & 0.9114 & 0.9019 \\
\hline Robot_LvF & 0.9986 & 0.9997 & 0.9913 & 0.9997 & 0.9987 & 0.9909 & 0.9986 & 0.9960 & 0.9984 & 0.9848 \\
\hline Robot_RvF & 0.9974 & 0.9922 & 0.9745 & 0.9923 & 0.9960 & 0.9966 & 0.9960 & 0.9881 & 0.9965 & 0.9882 \\
\hline Ecoli_OMvCP & 0.9203 & 0.8326 & 0.8993 & 0.8870 & 0.8659 & 0.9268 & 0.8993 & 0.8826 & 0.9080 & 0.9609 \\
\hline Ecoli_PPvCP & 0.9322 & 0.9199 & 0.8728 & 0.9406 & 0.9319 & 0.8895 & 0.9326 & 0.9362 & 0.9533 & 0.9232 \\
\hline Ecoli_IMvCP & 0.9882 & 0.9752 & 0.9563 & 0.9752 & 0.9795 & 0.9795 & 0.9839 & 0.9839 & 0.9795 & 0.9637 \\
\hline Glass_567vAll & 0.9735 & 0.9731 & 0.9957 & 0.9644 & 0.9692 & 0.9281 & 0.9727 & 0.9822 & 0.9909 & 0.9870 \\
\hline Vehicle_VANvAll & 0.9437 & 0.9339 & 0.9517 & 0.9429 & 0.9472 & 0.9307 & 0.9506 & 0.9490 & 0.9575 & 0.9426 \\
\hline Vehicle_OPELvAll & 0.8272 & 0.8314 & 0.8204 & 0.8276 & 0.8246 & 0.8333 & 0.8211 & 0.8561 & 0.8193 & 0.8322 \\
\hline Vehicle_ASSBvAll & 0.8424 & 0.8399 & 0.8021 & 0.8077 & 0.8313 & 0.8543 & 0.8345 & 0.8279 & 0.8417 & 0.8367 \\
\hline Vehicle_BUSvAll & 0.9515 & 0.9444 & 0.9293 & 0.9434 & 0.9410 & 0.9564 & 0.9508 & 0.9718 & 0.9632 & 0.9506 \\
\hline Wine_3vAll & 0.9462 & 0.9769 & 0.9669 & 0.9538 & 0.9385 & 0.9592 & 0.9462 & 0.9769 & 0.9538 & 0.9519 \\
\hline Wine_1vAll & 0.9380 & 0.9432 & 0.9771 & 0.9771 & 0.9380 & 0.9523 & 0.9575 & 0.9541 & 0.9470 & 0.9594 \\
\hline Wine_2vAll & 0.8898 & 0.8861 & 0.8963 & 0.8652 & 0.9401 & 0.8898 & 0.9460 & 0.9251 & 0.9096 & 0.9316 \\
\hline Breast-tissue_CFvAll & 0.8100 & 0.7600 & 0.7700 & 0.7500 & 0.7400 & 0.7400 & 0.7500 & 0.7500 & 0.7400 & 0.8500 \\
\hline Breast-cancer_MvB & 0.9323 & 0.9556 & 0.9292 & 0.9583 & 0.9011 & 0.9435 & 0.9414 & 0.9509 & 0.9509 & 0.9276 \\
\hline Ionosphere_BvG & 0.8594 & 0.8368 & 0.8283 & 0.8631 & 0.8705 & 0.8205 & 0.8632 & 0.8712 & 0.8629 & 0.8591 \\
\hline PageBlocks_4v2 & 0.9667 & 0.9611 & 0.9431 & 0.9667 & 0.9577 & 0.9778 & 0.9667 & 0.9688 & 0.9688 & 0.9661 \\
\hline PageBlocks_5v2 & 0.9830 & 0.9798 & 0.9662 & 0.9630 & 0.9662 & 0.9729 & 0.9662 & 0.9763 & 0.9660 & 0.9361 \\
\hline Segment_4v123 & 0.9728 & 0.9584 & 0.9637 & 0.9684 & 0.9731 & 0.9692 & 0.9647 & 0.9702 & 0.9738 & 0.9676 \\
\hline Segment_5v123 & 0.9458 & 0.9237 & 0.9214 & 0.9365 & 0.9437 & 0.9280 & 0.9380 & 0.9445 & 0.9378 & 0.9397 \\
\hline Segment_6v123 & 1.0000 & 1.0000 & 1.0000 & 1.0000 & 1.0000 & 1.0000 & 1.0000 & 1.0000 & 1.0000 & 1.0000 \\
\hline
\end{tabular}


Table 4. Comparison of F-measure among different oversampling techniques

\begin{tabular}{|c|c|c|c|c|c|c|c|c|c|c|}
\hline Dataset & $\overline{\mathrm{SM}}$ & Bor-1 & $\overline{\text { Bor-2 }}$ & $\overline{\mathrm{ADA}}$ & MWM & $\mathrm{ACO}$ & OTEMD-LLE-1 & OTEMD-LLE-2 & OTEMD-LLE-3 & OTEMD-LLE-4 \\
\hline Abalone_18v9 & 0.4374 & 0.4566 & 0.5858 & 0.5413 & 0.4704 & 0.5073 & 0.5058 & 0.4343 & 0.4865 & 0.5348 \\
\hline Abalone_17v7 & 0.9058 & 0.9081 & 0.8621 & 0.8901 & 0.9043 & 0.8738 & 0.9026 & 0.8899 & 0.8670 & 0.8652 \\
\hline Abalone_19v5 & 0.9703 & 0.9769 & 0.9846 & 0.9703 & 0.9923 & 0.9636 & 0.9846 & 0.9769 & 0.9769 & 0.9779 \\
\hline CTG_PvN & 0.9512 & 0.9177 & 0.8929 & 0.9188 & 0.9449 & 0.8429 & 0.9576 & 0.9365 & 0.9338 & 0.8888 \\
\hline CTG_SvN & 0.8727 & 0.8336 & 0.7585 & 0.8506 & 0.8801 & 0.7888 & 0.8836 & 0.8267 & 0.8715 & 0.7886 \\
\hline Statlandsat_4v12 & 0.9588 & 0.9616 & 0.9657 & 0.9673 & 0.9573 & 0.9643 & 0.9657 & 0.9614 & 0.9829 & 0.9775 \\
\hline Statlandsat_5v12 & 0.9353 & 0.9684 & 0.9086 & 0.9556 & 0.9297 & 0.9306 & 0.9560 & 0.9618 & 0.9635 & 0.9408 \\
\hline Libra_123vall & 0.8687 & 0.8751 & 0.8528 & 0.8689 & 0.8601 & 0.8714 & 0.8757 & 0.8574 & 0.8665 & 0.8331 \\
\hline Libra_456vAll & 0.9411 & 0.9386 & 0.9110 & 0.9414 & 0.9468 & 0.8904 & 0.9441 & 0.9162 & 0.9392 & 0.9039 \\
\hline Libra_789vAll & 0.9167 & 0.8605 & 0.8934 & 0.9230 & 0.9033 & 0.9001 & 0.9106 & 0.9215 & 0.9203 & 0.8912 \\
\hline Yeast_ME1vCYT & 0.9751 & 0.9815 & 0.9700 & 0.9772 & 0.9684 & 0.8984 & 0.9786 & 0.9575 & 0.9786 & 0.9623 \\
\hline Yeast_ME1vNUC & 0.9769 & 0.9550 & 0.9473 & 0.9769 & 0.9769 & 0.9340 & 0.9735 & 0.9846 & 0.9735 & 0.9684 \\
\hline Yeast_ME2vCYT & 0.9044 & 0.8856 & 0.7536 & 0.9002 & 0.8920 & 0.7667 & 0.8836 & 0.8929 & 0.9057 & 0.8553 \\
\hline Yeast_ME2vNUC & 0.9284 & 0.8835 & 0.7689 & 0.9157 & 0.9169 & 0.8120 & 0.9047 & 0.8938 & 0.8995 & 0.8396 \\
\hline Yeast_ME3vCYT & 0.9217 & 0.9123 & 0.8459 & 0.9258 & 0.9231 & 0.9096 & 0.9238 & 0.9393 & 0.9312 & 0.8840 \\
\hline Yeast_ME3vNUC & 0.9168 & 0.8954 & 0.8706 & 0.8992 & 0.9189 & 0.8939 & 0.8935 & 0.9116 & 0.9154 & 0.9054 \\
\hline Robot_LvF & 0.9948 & 0.9949 & 0.9501 & 0.9936 & 0.9889 & 0.9635 & $\begin{array}{l}0.9930 \\
0.9942\end{array}$ & 0.9515 & 0.9941 & 0.8847 \\
\hline Robot_RvF & 0.9879 & 0.9805 & 0.9352 & 0.9797 & 0.9895 & 0.9874 & 0.9882 & 0.9607 & 0.9835 & 0.9056 \\
\hline Ecoli_OMvCP & 0.7721 & 0.8535 & 0.8545 & 0.8324 & 0.8081 & 0.7657 & 0.8033 & 0.8163 & 0.8321 & 0.8394 \\
\hline Ecoli_IMvAll & 0.9556 & 0.9693 & 0.9275 & 0.9423 & 0.9668 & 0.9504 & 0.9640 & 0.9697 & 0.9557 & 0.9286 \\
\hline Ecoli_PPvCP & 0.8902 & 0.9081 & 0.8754 & 0.8747 & 0.9298 & 0.8540 & 0.9281 & 0.9401 & 0.9251 & 0.8859 \\
\hline Ecoli_IMvCP & 0.9825 & 0.9913 & 0.963 & 0.9631 & 0.9854 & 0.9715 & 0.9882 & 0.9831 & 0.9773 & 0.9587 \\
\hline Glass_567vAll & 0.9631 & 0.9821 & 0.9731 & 0.9778 & 0.9861 & 0.9603 & $\begin{array}{l}0.9639 \\
0.9639\end{array}$ & 0.9492 & 0.9492 & 0.9268 \\
\hline Vehicle_VANvAll & 0.9117 & 0.9204 & 0.9016 & 0.9209 & 0.9174 & 0.9188 & 0.9129 & 0.9128 & 0.9142 & 0.9238 \\
\hline Vehicle_OPELvAll & 0.8135 & 0.7669 & 0.7934 & 0.7973 & 0.8048 & 0.8504 & 0.7867 & 0.7785 & 0.8026 & 0.8286 \\
\hline Vehicle_ASSBvall & 0.8305 & 0.7958 & 0.8067 & 0.7928 & 0.8143 & 0.8940 & 0.8076 & 0.8179 & 0.8154 & 0.8286 \\
\hline Vehicle_BUSvAll & 0.9643 & 0.9591 & 0.9553 & 0.9663 & 0.9619 & 0.9696 & 0.9603 & 0.9606 & 0.9629 & 0.9547 \\
\hline Wine_3vAll & 0.9720 & 0.9408 & 0.9539 & 0.9615 & 0.9679 & 0.9190 & 0.9606 & 0.9763 & 0.9646 & 0.9532 \\
\hline Wine_1vAll & 0.9706 & 0.9600 & 0.9484 & 0.9706 & 0.9561 & 0.9343 & 0.9632 & 0.9674 & 0.9534 & 0.9445 \\
\hline Wine_2vAll & 0.8920 & 0.8955 & 0.8809 & 0.8729 & 0.9099 & 0.8556 & 0.8921 & 0.8935 & 0.8967 & 0.8816 \\
\hline Breast-tissue_CFvall & 0.7233 & 0.7297 & 0.7197 & 0.7588 & 0.7490 & 0.7430 & 0.7962 & 0.7191 & 0.7842 & 0.7751 \\
\hline Breast-cancer_MvB & 0.9065 & 0.9085 & 0.8903 & 0.8995 & 0.8979 & 0.9263 & 0.9086 & 0.9136 & 0.9118 & 0.9051 \\
\hline Ionosphere_BvG & 0.8615 & 0.8598 & 0.8585 & 0.8403 & 0.8638 & 0.8707 & 0.8562 & 0.8416 & 0.8429 & 0.8274 \\
\hline PageBlocks_4v2 & 0.9748 & 0.9625 & 0.9487 & 0.9686 & 0.9601 & 0.9598 & 0.9663 & 0.9598 & 0.9717 & 0.9327 \\
\hline PageBlocks_5v2 & 0.9671 & 0.9805 & 0.9257 & 0.9724 & 0.9629 & 0.9802 & 0.9604 & 0.9709 & 0.9694 & 0.9834 \\
\hline Segment_4v123 & 0.9650 & 0.9592 & 0.9449 & 0.9675 & 0.9695 & 0.9589 & 0.9640 & 0.9606 & $\begin{array}{l}0.9694 \\
0.968\end{array}$ & $\begin{array}{l}0.90327 \\
0.9327\end{array}$ \\
\hline Segment_5v123 & 0.9194 & 0.9014 & 0.8918 & 0.9142 & 0.9155 & 0.9006 & 0.9188 & 0.9173 & 0.9194 & 0.9017 \\
\hline Segment_6v123 & 1.0000 & 1.0000 & 1.0000 & 1.0000 & 1.0000 & 1.0000 & 1.0000 & 1.0000 & 1.0000 & 0.9988 \\
\hline Segment_7v 123 & 0.9950 & 0.9981 & 0.9962 & 0.9968 & 0.9975 & 0.9944 & 0.9944 & 0.9931 & 0.9950 & 0.9908 \\
\hline average value & 0.9059 & 0.8965 & 0.8980 & 0.9122 & 0.9055 & 0.8931 & 0.9203 & 0.9002 & 0.9138 & 0.8919 \\
\hline
\end{tabular}

Table 5. Comparison of G-meam among different oversampling techniques

\begin{tabular}{|c|c|c|c|c|c|c|c|c|c|c|}
\hline Dataset & SM & Bor-1 & Bor-2 & $\mathrm{ADA}$ & MWM & $\mathrm{ACO}$ & OTEMD-LLE-1 & OTEMD-LLE-2 & OTEMD-LLE-3 & OTEMD-LLE-4 \\
\hline Abalone_18v9 & 0.5447 & 0.4081 & 0.5304 & 0.4600 & 0.4268 & 0.4594 & 0.4056 & 0.4098 & 0.4826 & 0.4938 \\
\hline Abalone_17v7 & 0.9236 & 0.8954 & 0.8884 & 0.9036 & 0.9032 & 0.8580 & 0.8714 & 0.9018 & 0.8911 & 0.9026 \\
\hline Abalone_19v5 & 0.9703 & 0.9626 & 0.9692 & 0.9692 & 0.9703 & 0.9779 & 0.9703 & 0.9626 & 0.9626 & 0.9626 \\
\hline CTG_PvN & 0.9662 & 0.9137 & 0.9009 & 0.9320 & 0.9444 & 0.8227 & 0.9589 & 0.9665 & 0.9483 & 0.9259 \\
\hline CTG_SvN & 0.8801 & 0.8480 & 0.7780 & 0.8489 & 0.8736 & 0.8041 & 0.8902 & 0.8508 & 0.8585 & 0.7976 \\
\hline Statlandsat_4v12 & 0.9528 & 0.9515 & 0.9620 & 0.9557 & 0.9546 & 0.9407 & 0.9601 & 0.9528 & 0.9700 & 0.9699 \\
\hline Statlandsat_5v12 & 0.9363 & 0.9724 & 0.9099 & 0.9697 & 0.9363 & 0.9341 & 0.9433 & 0.9486 & 0.9487 & 0.9401 \\
\hline Libra_123vAll & 0.8621 & 0.8717 & 0.8430 & 0.8724 & 0.8548 & 0.8327 & 0.8645 & 0.8562 & 0.8498 & 0.8653 \\
\hline Libra_456vAll & 0.9417 & 0.9383 & 0.9238 & 0.9390 & 0.9442 & 0.8955 & 0.9250 & 0.9149 & 0.9077 & 0.9171 \\
\hline Libra_789vall & 0.9279 & 0.9089 & 0.9218 & 0.9219 & 0.9240 & 0.9006 & 0.9213 & 0.9146 & 0.9320 & 0.9040 \\
\hline Yeast_ME1vCYT & 0.9714 & 0.9666 & 0.9118 & 0.9748 & 0.9783 & 0.9070 & 0.9783 & 0.9714 & 0.9783 & 0.9679 \\
\hline Yeast_ME1vNUC & 0.9503 & 0.9586 & 0.9567 & 0.9572 & 0.9632 & 0.9210 & 0.9494 & 0.9609 & 0.9535 & 0.9585 \\
\hline Yeast_ME2vCYT & 0.9061 & 0.9162 & 0.7472 & 0.9417 & 0.9281 & 0.8596 & 0.9003 & 0.9075 & 0.9203 & 0.8464 \\
\hline Yeast_ME3vCYT & 0.9191 & 0.9136 & 0.8749 & 0.9102 & 0.9103 & 0.9131 & 0.9204 & 0.9221 & 0.9207 & 0.8899 \\
\hline Yeast_ME3vNUC & 0.8921 & 0.8955 & 0.8526 & 0.8980 & 0.8979 & 0.9039 & 0.9084 & 0.9070 & 0.9045 & 0.8974 \\
\hline Robot_LvF & 0.9974 & 0.9981 & 0.9484 & 0.9968 & 0.9974 & 0.9523 & 0.9955 & 0.9608 & 0.9968 & 0.8756 \\
\hline Robot_RvF & 0.9855 & 0.9763 & 0.9341 & 0.9827 & 0.9850 & 0.9871 & 0.9924 & 0.9612 & 0.9843 & 0.9402 \\
\hline Ecoli_OMvCP & 0.8659 & 0.8659 & 0.8302 & 0.8576 & 0.8911 & 0.8653 & 0.8632 & 0.8802 & 0.8788 & 0.8565 \\
\hline Ecoli_IMvAll & 0.9556 & 0.9756 & 0.9738 & 0.9469 & 0.9613 & 0.9627 & 0.9729 & 0.9820 & 0.9670 & 0.9417 \\
\hline Ecoli_PPvCP & 0.8949 & 0.8559 & 0.8161 & 0.8364 & 0.8566 & 0.8548 & 0.8816 & 0.8735 & 0.8911 & 0.8437 \\
\hline Ecoli_IMvCP & 0.9675 & 0.9759 & 0.9539 & 0.9561 & 0.9710 & 0.9637 & 0.9823 & 0.9768 & 0.9823 & 0.9545 \\
\hline Glass_567vAll & 0.9564 & 0.9842 & 0.9458 & 0.9957 & 0.9870 & 0.9353 & 0.9660 & 0.9660 & 0.9617 & 0.9624 \\
\hline Vehicle_VANvAll & 0.9226 & 0.9365 & 0.9242 & 0.9406 & 0.9356 & 0.9432 & 0.9216 & 0.9408 & 0.9274 & 0.9457 \\
\hline Vehicle_OPELvAll & 0.7811 & 0.7810 & 0.7819 & 0.7882 & 0.7843 & 0.8636 & 0.7458 & 0.7811 & 0.8071 & 0.7903 \\
\hline Vehicle_ASSBvAll & 0.8137 & 0.7769 & 0.8079 & 0.7819 & 0.7858 & 0.8880 & 0.8189 & 0.8030 & 0.7814 & 0.8098 \\
\hline Vehicle_BUSvAll & 0.9610 & 0.9446 & 0.9492 & 0.9581 & 0.9489 & 0.9520 & 0.9471 & 0.9461 & 0.9475 & 0.9253 \\
\hline Wine_1vAll & 0.9754 & 0.9657 & 0.9466 & 0.9791 & 0.9652 & 0.9332 & 0.9625 & 0.9754 & 0.9721 & 0.9721 \\
\hline Wine_2vAll & 0.8917 & 0.9014 & 0.8892 & 0.8994 & 0.9079 & 0.8977 & 0.9261 & 0.9113 & 0.9084 & 0.9000 \\
\hline Breast-tissue_CFvall & 0.7587 & 0.7083 & 0.7315 & 0.7436 & 0.7262 & 0.7628 & 0.7643 & 0.7616 & 0.8122 & 0.7599 \\
\hline Breast-cancer_MvB & 0.9224 & 0.9182 & 0.9186 & 0.9360 & 0.9268 & 0.9171 & 0.9169 & 0.9141 & 0.9189 & 0.9188 \\
\hline Ionosphere_BvG & 0.8711 & 0.8546 & 0.8446 & 0.8535 & 0.8685 & 0.8532 & 0.8616 & 0.8550 & 0.8617 & 0.8453 \\
\hline PageBlocks_4v2 & 0.9635 & 0.9539 & 0.9464 & 0.9628 & 0.9576 & 0.9621 & 0.9547 & 0.9606 & 0.9636 & 0.9610 \\
\hline PageBlocks_5v2 & 0.9662 & 0.9756 & 0.9728 & 0.9657 & 0.9726 & 0.9666 & 0.9632 & 0.9613 & 0.9595 & 0.9640 \\
\hline Segment_4v123 & 0.9706 & 0.9611 & 0.9532 & 0.9672 & 0.9669 & 0.9426 & 0.9629 & 0.9646 & 0.9651 & 0.9505 \\
\hline Segment_5v123 & 0.9269 & 0.9111 & 0.8907 & 0.9163 & 0.9112 & 0.8933 & 0.9248 & 0.9099 & 0.9164 & 0.9039 \\
\hline Segment_6v123 & 1.0000 & 1.0000 & 1.0000 & 1.0000 & 1.0000 & 1.0000 & 1.0000 & 1.0000 & 1.0000 & 0.9994 \\
\hline Segment_7v123 & 0.9969 & 0.9944 & 0.9957 & 0.9957 & 0.9957 & 0.9931 & 0.9950 & 0.9963 & 0.9951 & 0.9944 \\
\hline average value & 0.9200 & 0.9145 & 0.8981 & 0.9200 & 0.9218 & 0.9091 & 0.9236 & 0.9210 & 0.9269 & 0.9087 \\
\hline
\end{tabular}


Table 6. The performances of OTEMD with various mapping methods

\begin{tabular}{c|l|cccc}
\hline Algorithm & Metric & OS1 & OS2 & OS3 & OS4 \\
\hline \multirow{3}{*}{ HLLE } & AUC & 0.9343 & 0.9355 & 0.9316 & 0.9243 \\
& F-measure & 0.9064 & 0.9056 & 0.9183 & 0.8896 \\
& G-mean & 0.9245 & 0.9016 & 0.9134 & 0.9200 \\
\hline \multirow{3}{*}{ MLLE } & AUC & 0.9341 & 0.9344 & 0.9320 & 0.9316 \\
& F-measure & 0.9023 & 0.9039 & 0.9060 & 0.8946 \\
& G-mean & 0.9215 & 0.9188 & 0.9145 & 0.9245 \\
\hline \multirow{3}{*}{ ATSA } & AUC & 0.9372 & 0.9368 & 0.9341 & 0.9262 \\
& F-measure & 0.9156 & 0.9064 & 0.8912 & 0.9056 \\
& G-mean & 0.9113 & 0.8997 & 0.9245 & 0.9212 \\
\hline
\end{tabular}

$p<5 \%$ means that the experimental results provide the significant evidence against $H_{0}$. Therefore, we reject the null hypothesis and accept the alternative hypothesis. By taking win-lose-draw as the evaluation criterion, a win method is better than the others at the $95 \%$ confidence level. The performances of the proposed oversampling techniques are compared with six traditional data-level strategies, as shown in Table 7 . The statistical results show that LLE-based OTEMD has the better and more stable classification performances, except for OTEMDLLE-4. By analysis, a minority sample generated by $O S_{4}$ based on the minority-minority or minority-majority matched-pair data may be mislabeled, resulting in the wrong classification boundary. In addition, the Wilcoxon paired sign-rank comparison listed in Table 8 also shows that OTEMD-LLE can capture the groundtruth structure of the dataset accurately and generate the valuable minority samples, thus, improving the classification performance in most of the cases.

\section{Conclusion}

To address the class imbalance problems, a novel oversampling strategy based on manifold distance is proposed, in which a new minority sample is generated in terms of the distances among neighbors in manifold space. The redundant majority data are firstly removed by undersampling to decrease the computation complexity of calculating the manifold distance among the samples. Thereafter, the nearest ones are chosen as neighbors and form the matched-pair data. Based on them, four improved oversampling strategies are presented to create a new minority sample nearby the classification boundary. The experiments on the 39 UCI datasets and 9 synthetic datasets indicate that OTEMD has better classification accuracy in most cases, especially for the datasets with class overlapping and small disjunction. Moreover, the oversampling strategies have more significant effect on OTEMD performance. The proposed oversampling technique provides a generic framework for data-based class imbalance learning. The combination of advanced optimizing techniques (Guo et al., 2020, 2019) with OTEMD is our future work. Moreover, it's interesting to investigate more efficient manifold learning methods for class imbalance learning.

\section{Acknowledgement}

This work was jointly supported by National Natural Science Foundation of China (61973305, 61573361 and 61803369), and Natural Science Foundation of Liaoning Province for the State Key Laboratory of Robotics(2020KF-22-02). Also, thank you for the support from the State Key Laboratory of Robotics (2019-O12).

\section{References}

Arafat, Y., Hoque, S., Xu, S. and Farid, D. M. (2019) 'Machine learning for mining imbalanced data.', IAENG Internaitonal journal of computer science, Vol. 46, pp.332-348

Barua, S., Islam, M. M., Yao, X. and Murase, K. (2013) 'Mwmote-majority weighted minority oversampling technique for imbalanced data set learning.', IEEE Transactions on Knowledge and Data Engineering, Vol. 26, No. 2, pp.405-425

Bellinger, C., Drummond, C. and Japkowicz, N. (2017) 'Manifold-based synthetic oversampling with manifold conformance estimation.', Machine Learning, Vol. 107, No. 1, pp.605-637

Cai, B., Zhao, Y., Liu, H. and Min, X. (2017) 'A datadriven fault diagnosis methodology in three-phase inverters for pmsm drive systems.', IEEE Transactions on Power Electronics, Vol. 32, No. 99, pp.5590-5600

Cheng, J. , Chen, J. , Guo, Y. N. , Cheng, S. , Yang, L. , and Zhang, P. (2018) 'Adaptive CCR-ELM with variable-length brain storm optimization algorithm for class-imbalance learning.', Natural Computing,[online] https://doi.org/10.1007/s11047-019-09735-9

Dal, P. A., Boracchi, G., Caelen, O., Alippi, C. and Bontempi, G. (2018) 'Credit card fraud detection: A realistic modeling and a novel learning strategy.', IEEE Transactions on Neural Networks and Learning Systems, Vol. 29, No. 8, pp.3784-3797

Guo, Y., Zhang, X., Gong, D.-W., Zhang, Z. and Yang, J.-J. (2020) 'Novel interactive preferencebased multi-objective evolutionary optimization for bolt supporting networks.', IEEE Transactions on Evolutionary Computation, Vol. 24, No. 4, pp.750-764

Guo, Y., Yang, H., Chen, M., Cheng, J. and Gong, D. (2019) 'Ensemble prediction-based dynamic robust multi-objective optimization methods.', Swarm and Evolutionary Computation, Vol. 48, pp.156-171

Han, H., Wang, W. Y. and Mao, B. H. (2005) 'Borderline-smote: A new over-sampling method in imbalanced data sets learning.', In Advances in Intelligent Computing, pp.878-887 
Table 7. Win-Lose-Draw results of Wilcoxon pairwise test

\begin{tabular}{ccccccc}
\hline & SM & Bor-1 & Bor-2 & ADA & MWM & ACO \\
\hline OTEMD-LLE-1 & $3-8-28$ & $9-4-26$ & $16-2-21$ & $6-5-28$ & $6-3-30$ & $10-4-25$ \\
OTEMD-LLE-2 & $9-8-22$ & $9-6-24$ & $15-4-20$ & $7-8-24$ & $10-6-23$ & $13-6-20$ \\
OTEMD-LLE-3 & $7-6-26$ & $11-7-21$ & $15-4-20$ & $12-7-20$ & $9-2-28$ & $9-3-27$ \\
OTEMD-LLE-4 & $8-11-20$ & $10-13-16$ & $14-7-18$ & $10-14-15$ & $7-10-22$ & $10-11-18$ \\
OTEMD-HLLE-1 & $2-4-33$ & $11-4-24$ & $15-1-23$ & $10-6-23$ & $4-4-31$ & $13-4-22$ \\
OTEMD-HLLE-2 & $4-8-27$ & $10-10-19$ & $15-3-21$ & $9-8-22$ & $4-7-28$ & $10-10-19$ \\
OTEMD-HLLE-3 & $2-4-33$ & $11-5-23$ & $10-2-27$ & $8-10-21$ & $4-10-25$ & $10-7-22$ \\
OTEMD-HLLE-4 & $1-16-22$ & $8-16-15$ & $10-10-19$ & $5-17-17$ & $3-17-19$ & $5-13-21$ \\
OTEMD-MLLE-1 & $3-6-30$ & $10-0-29$ & $15-4-20$ & $7-10-22$ & $7-6-26$ & $9-6-24$ \\
OTEMD-MLLE-2 & $5-4-30$ & $8-0-31$ & $16-2-21$ & $5-7-27$ & $9-4-26$ & $9-7-23$ \\
OTEMD-MLLE-3 & $3-4-32$ & $13-2-24$ & $18-2-19$ & $10-4-25$ & $7-3-29$ & $8-6-25$ \\
OTEMD-MLLE-4 & $3-8-28$ & $7-5-27$ & $13-4-22$, & $4-8-27$, & $4-9-26$, & $9-11-19$ \\
OTEMD-LTSA-1 & $7-5-27$ & $14-2-23$ & $21-1-17$ & $9-7-23$ & $9-6-24$ & $11-3-25$ \\
OTEMD-LTSA-2 & $5-9-25$ & $11-7-21$ & $16-1-22$ & $5-6-28$ & $7-9-23$ & $10-8-21$ \\
OTEMD-LTSA-3 & $4-6-29$ & $12-5-22$ & $14-0-25$ & $9-5-25$ & $3-6-30$ & $9-5-25$ \\
OTEMD-LTSA-4 & $2-14-23$ & $5-9-25$ & $9-5-25$ & $2-15-22$ & $4-15-20$ & $5-11-23$ \\
\hline
\end{tabular}

Table 8. Significance Test of Averaged AUC between OTEMD-LLE-1, -2, -3, -4 and MWMOTE

\begin{tabular}{|c|c|c|c|c|c|c|c|c|c|c|c|c|c|}
\hline \multirow[t]{2}{*}{ Dataset } & \multirow[t]{2}{*}{ MWMOTE } & \multicolumn{3}{|c|}{ OTEMD-LLE-1 vs. MWM } & \multicolumn{3}{|c|}{ OTEMD-LLE-2 vs. MWM } & \multicolumn{3}{|c|}{ OTEMD-LLE-3 vs. MWM } & \multicolumn{3}{|c|}{ OTEMD-LLE-4 vs. MWM } \\
\hline & & OTEMD & $\mathrm{R}+$ & $\mathrm{R}-$ & OTEMD & $\mathrm{R}+$ & $\mathrm{R}-$ & OTEMD & $\mathrm{R}+$ & $\mathrm{R}-$ & OTEMD & $\mathrm{R}+$ & $\mathrm{R}-$ \\
\hline Abalone_18v9 & 0.625 & 0.6608 & 38 & - & 0.7346 & 39 & - & 0.657 & 35 & - & 0.65 & 31 & - \\
\hline Abalone_17v7 & 0.9409 & 0.9287 & - & 30 & 0.9089 & - & 35 & 0.8794 & - & 39 & 0.9133 & - & 33 \\
\hline Abalone_19v5 & 0.9866 & 0.9933 & 17 & - & 0.9933 & 15 & - & 0.9933 & 15 & - & 0.9933 & 9 & - \\
\hline CTG_PvN & 0.9649 & 0.956 & - & 25 & 0.9721 & 17 & - & 0.9708 & 12 & - & 0.9825 & 27 & - \\
\hline CTG_SvN & 0.9312 & 0.9544 & 35 & - & 0.9521 & 29 & - & 0.9502 & 29 & - & 0.9466 & 22 & - \\
\hline Statlandsat_4v12 & 0.9774 & 0.9845 & 18 & - & 0.9816 & 11 & - & 0.976 & - & 9 & 0.9802 & 3 & - \\
\hline Statlandsat_5v12 & 0.968 & 0.9688 & 7 & - & 0.9702 & 7 & - & 0.9588 & - & 20 & 0.9783 & 17 & - \\
\hline Libra_123vAll & 0.9069 & 0.9122 & 14 & - & 0.9055 & - & 4 & 0.9258 & 28 & - & 0.871 & - & 37 \\
\hline Libra_456vAll & 0.9693 & 0.9543 & - & 31 & 0.9504 & - & 27 & 0.9301 & - & 36 & 0.9361 & - & 35 \\
\hline Libra_789vAll & 0.9421 & 0.9523 & 29 & - & 0.9248 & - & 26 & 0.955 & 24 & - & 0.9069 & - & 36 \\
\hline Yeast_ME1vCYT & 0.9769 & 0.9928 & 32 & - & 0.9984 & 30 & - & 0.9928 & 26 & - & 0.9928 & 24 & - \\
\hline Yeast_ME1vNUC & 0.9714 & 0.9785 & 19 & - & 0.9785 & 16 & - & 0.9785 & 16 & - & 0.9908 & 29 & - \\
\hline Yeast_ME2vCYT & 0.9782 & 0.9616 & - & 33 & 0.9252 & - & 38 & 0.9722 & - & 13 & 0.9766 & - & 2 \\
\hline Yeast_ME2vNUC & 0.9372 & 0.9645 & 36 & - & 0.9666 & 32 & - & 0.9554 & 27 & - & 0.9513 & 21 & - \\
\hline Yeast_ME3vCYT & 0.9378 & 0.9454 & 21 & - & 0.9623 & 31 & - & 0.9584 & 30 & - & 0.9207 & - & 25 \\
\hline Yeast_ME3vCYT & 0.9189 & 0.9113 & - & 22 & 0.8985 & - & 28 & 0.9113 & - & 18 & 0.9018 & - & 26 \\
\hline Robot_LvF & 0.9987 & 0.9985 & - & 5 & 0.996 & - & 8 & 0.9984 & - & 6 & 0.9848 & - & 20 \\
\hline Robot_RvF & 0.996 & 0.996 & 4 & 2.0 & 0.988 & - & 18 & 0.9964 & 7 & - & 0.9882 & - & 11 \\
\hline Ecoli_IMvAll & 0.9772 & 0.9772 & 0.5 & 0.5 & 0.9823 & 14 & - & 0.9797 & 10 & - & 0.9684 & - & 15 \\
\hline Ecoli_PPvCP & 0.9318 & 0.9326 & 6 & - & 0.9362 & 12 & - & 0.9532 & 31 & - & 0.9231 & - & 14 \\
\hline Ecoli_IMvCP & 0.9795 & 0.9838 & 13 & - & 0.9838 & 13 & - & 0.9795 & 1.0 & 1.0 & 0.9636 & - & 23 \\
\hline Glass_567vAll & 0.9691 & 0.9727 & 12 & - & 0.9822 & 22 & - & 0.9909 & 32 & - & 0.9869 & 28 & - \\
\hline Vehicle_VANvAll & 0.9471 & 0.9506 & 10 & - & 0.949 & 5 & - & 0.9575 & 21 & - & 0.9426 & - & 6 \\
\hline Vehicle_OPELvAll & 0.8246 & 0.8211 & - & 11 & 0.856 & 34 & - & 0.8193 & - & 11 & 0.8321 & 10 & - \\
\hline Vehicle_ASSBvAll & 0.8313 & 0.8344 & 9 & - & 0.8278 & - & 10 & 0.8416 & 22 & - & 0.8367 & 7 & - \\
\hline Vehicle_BUSvAll & 0.9409 & 0.9507 & 27 & - & 0.9718 & 33 & - & 0.9631 & 33 & - & 0.9505 & 16 & - \\
\hline Wine_3vAll & 0.9384 & 0.9461 & 23 & - & 0.9769 & 36 & - & 0.9538 & 25 & - & 0.9519 & 19 & - \\
\hline Wine_1vAll & 0.9379 & 0.9575 & 34 & - & 0.9541 & 24 & - & 0.9469 & 19 & - & 0.9593 & 30 & - \\
\hline Wine_2vAll & 0.9401 & 0.9459 & 16 & - & 0.9251 & - & 23 & 0.9096 & - & 34 & 0.9315 & - & 13 \\
\hline Breast-tissue_CFvAll & 0.74 & 0.75 & 28 & - & 0.75 & 19 & - & 0.74 & 2.0 & 2.0 & 0.85 & 39 & - \\
\hline Breast-cancer_MvB & 0.9011 & 0.9413 & 39 & - & 0.9508 & 37 & - & 0.9508 & 38 & - & 0.9275 & 32 & - \\
\hline Ionosphere_BvG & 0.8704 & 0.8632 & - & 20 & 0.8712 & 3 & - & 0.8629 & - & 17 & 0.859 & - & 18 \\
\hline PageBlocks_4v2 & 0.9576 & 0.9666 & 26 & - & 0.9687 & 21 & - & 0.9687 & 23 & - & 0.966 & 12 & - \\
\hline PageBlocks_5v2 & 0.9662 & 0.9662 & 1.0 & 1.0 & 0.9763 & 20 & - & 0.9659 & - & 5 & 0.936 & - & 34 \\
\hline Segment_4v123 & 0.973 & 0.9647 & - & 24 & 0.9701 & - & 9 & 0.9738 & 8 & - & 0.9676 & - & 8 \\
\hline Segment_5v123 & 0.9437 & 0.9379 & - & 15 & 0.9444 & 2 & - & 0.9377 & - & 14 & 0.9396 & - & 5 \\
\hline Segment_7v123 & & \multicolumn{3}{|c|}{$p_{\text {value }}=0.0256$} & \multicolumn{3}{|c|}{$p_{\text {value }}=0.0290$} & \multicolumn{3}{|c|}{$p_{\text {value }}=0.0209$} & \multicolumn{3}{|c|}{$p_{\text {value }}=0.7223$} \\
\hline
\end{tabular}


He, H., Yang, B., Garcia, E. A. and Li, S. (2008) 'Adasyn: Adaptive synthetic sampling approach for imbalanced learning', In IEEE International Joint Conference on Neural Networks, pp.1322-1328

Hsu, C. C., Wang, K. S. and Chang, S. H. (2011) 'Bayesian decision theory for support vector machines: Imbalance measurement and feature optimization.', Expert Systems with Applications, Vol. 38, No. 5, pp.4698-4704

Kang, Q., Shi, L., Zhou, M. C., Wang, X. S., Wu, Q. D. and Wei, Z. (2018) 'A distance-based weighted undersampling scheme for support vector machines and its application to imbalanced classification.', IEEE Transactions on Neural Networks and Learning Systems, Vol. 29, pp.4152-4165

Kang, Q., Wang, K., Huang, B. and An, J. (2014) 'Kernel optimisation for kpca based on gaussianity estimation.', International Journal of Bio Inspired Computation, Vol. 6, No. 2, pp.91-107

Krawczyk, B. (2016) 'Learning from imbalanced data: open challenges and future directions.', Progress in Artificial Intelligence, Vol. 5, No. 4, pp.221-232

Lim, P., Goh, C. K. and Tan, K. C. (2016) 'Evolutionary cluster-based synthetic oversampling ensemble (ecoensemble) for imbalance learning.', IEEE Transactions on Cybernetics, Vol. 47, No. 99, pp.2850-2861

Lin, W. C., Tsai, C. F., Hu, Y. H. and Jhang, J. S. (2017) 'Clustering-based undersampling in class-imbalanced data.', Information Sciences, Vol. 409, pp.17-26

Loezer, L., Enembreck, F., Barddal, J. P. and de Souza Britto, A. (2020) 'Cost-sensitive learning for imbalanced data streams.', In The 35th ACM/SIGAPP Symposium on Applied Computing, pp. $498-504$

Lunga, D., Prasad, S., Crawford, M. M. and Ersoy, O. (2013) 'Manifold-learning-based feature extraction for classification of hyperspectral data: A review of advances in manifold learning.', IEEE Signal Processing Magazine, Vol. 31, No. 1, pp.55-66

Roweis, S. T. and Saul, L. K. (2000) 'Nonlinear dimensionality reduction by locally linear embedding.', Science, Vol. 290, No. 5500, pp.23232326

Shakeel, F., Sabhitha, A. S. and Sharma, S. (2017) 'Exploratory review on class imbalance problem: An overview.', In 2017 8th International Conference on Computing, Communication and Networking Technologies (ICCCNT), pp.1-8

Shen, Y., Wang, G. and Gao, H. (2016) 'Data-driven process monitoring based on modified orthogonal projections to latent structures.', IEEE Transactions on Control Systems Technology, Vol. 24, No. 4, pp.1480-1487
Soleymani, R., Granger, E. and Fumera, G. (2018) 'Progressive boosting for class imbalance and its application to face re-identification.', Expert Systems with Applications, Vol. 101, pp.271-291

Wang, Z., Xing, H., Li, T., Yan, Y. and Yi, P. (2016) 'A modified ant colony optimization algorithm for network coding resource minimization.', IEEE Transactions on Evolutionary Computation, Vol. 20, No. 3, pp.325-342

Zhang, S., Ma, Z. and Tan, H. (2018) 'On the equivalence of hlle and ltsa.', IEEE Transactions on Cybernetics, Vol. 48, No. 99, pp.742-753 